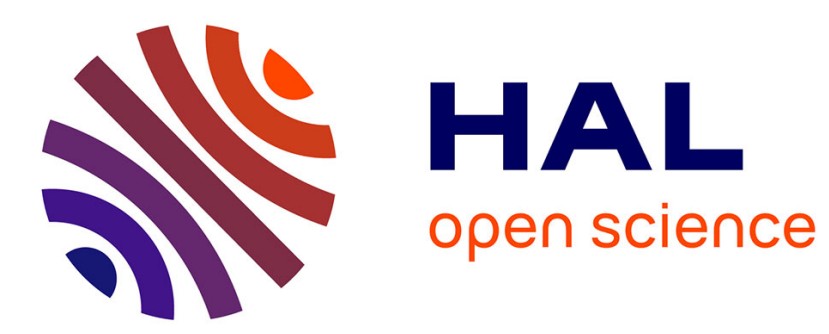

\title{
Méthodes d'étude du phénomène d'ionoluminescence atomique sur des cibles polycristallines minces
}

\author{
C. Schneider, J.P. Meriaux, R. Goutte, C. Guillaud
}

\section{To cite this version:}

C. Schneider, J.P. Meriaux, R. Goutte, C. Guillaud. Méthodes d'étude du phénomène d'ionoluminescence atomique sur des cibles polycristallines minces. Revue de Physique Appliquée, 1973, 8 (3), pp.283-288. 10.1051/rphysap:0197300803028300 . jpa-00243684

\section{HAL Id: jpa-00243684 https://hal.science/jpa-00243684}

Submitted on 1 Jan 1973

HAL is a multi-disciplinary open access archive for the deposit and dissemination of scientific research documents, whether they are published or not. The documents may come from teaching and research institutions in France or abroad, or from public or private research centers.
L'archive ouverte pluridisciplinaire HAL, est destinée au dépôt et à la diffusion de documents scientifiques de niveau recherche, publiés ou non, émanant des établissements d'enseignement et de recherche français ou étrangers, des laboratoires publics ou privés. 


\title{
MÉTHODES D'ÉTUDE DU PHÉNOMẼNE D'IONOLUMINESCENCE ATOMIQUE SUR DES CIBLES POLYCRISTALLINES MINCES
}

\author{
C. SCHNEIDER, J. P. MERIAUX, R. GOUTTE et C. GUILlAUD \\ Laboratoire d'Optique Corpusculaire et d'Ultrasons, \\ INSA, 69621 Villeurbanne, France
}

(Reçu le 21 février 1973)

\begin{abstract}
Résumé. - Le bombardement d'une feuille mince d'aluminium, d'épaisseur inférieure à $1000 \AA$ par un faisceau d'ions positifs de $20 \mathrm{keV}$ provoque, de part et d'autre de celle-ci, l'émission d'atomes excités caractéristiques respectivement du faisceau primaire et de la cible.

Après avoir décrit la méthode de préparation et de mesure de l'épaisseur des cibles minces, nous présentons deux dispositifs expérimentaux (photographique et spectroscopique) permettant d'étudier l'émission de lumière de chaque côté de la cible provoquée par la désexcitation des atomes excités.

Nous illustrons les possibilités de ces deux dispositifs par des exemples mettant en évidence les
\end{abstract} propriétés essentielles de ce phénomène émissif.

\begin{abstract}
The impact of $20 \mathrm{keV}$ ions on an aluminium foil (thickness less than $1000 \AA$ ) produces, on both sides of the target, the emission of excited atoms coming respectively from the ion beam and the target.

First the method of preparation and thickness measurement of thin foils are described ; then two experimental apparatuses - photographic and spectroscopic — are presented in order to study, on both sides of the thin target, the light emission resulting from the desexcitation of the excited atoms.

Some examples show the possibilities of these two apparatuses, and the essential characteristics of this emission phenomenon.
\end{abstract}

1. Introduction. - L'impact d'un faisceau d'ions positifs de quelques $\mathrm{keV}$ sur une surface solide provoque, en particulier, l'émission de particules excitées (atomes et ions provenant de la cible et du faisceau ionique incident). Le spectre de la lumière émise au voisinage de la surface bombardée est un spectre de raies produit par la désexcitation de toutes ces particules émises à l'état excité.

Dans le cas d'échantillons massifs polycristallins une étude spectrographique de ce phénomène de luminescence a été effectuée dans notre laboratoire [1] En particulier, la mesure des vitesses des atomes excités ainsi que la détermination des répartitions angulaires correspondantes ont permis de préciser le mécanisme d'émission de ces particules et de relier ce phénomène émissif aux autres phénomènes mieux connus que sont la pulvérisation cathodique et l'émission ionique secondaire.

Il nous a paru intéressant de reprendre cette étude dans le cas des couches polycristallines minces, en mettant à profit la possibilité d'observation simultanée des émissions lumineuses de chaque côté de la cible. En effet, dans ce cas, l'épaisseur de la couche constitue un paramètre supplémentaire important dont la variation permet de réaliser de nouvelles conditions d'excitation et de généraliser les résultats précédents.

Dans ce travail, nous décrivons essentiellement les méthodes d'observation du phénomène de luminescence dans le cas des couches minces et nous présentons quelques résultats illustrant les possibilités ainsi offertes.

2. Préparation des couches minces. - Pour ces premiers essais nous avons choisi l'aluminium comme élément constituant la cible, à cause du rendement élevé en ionoluminescence et du spectre très simple de ce métal.

Les couches ont été préparées par évaporation de l'aluminium sous vide sur des monocristaux de $\mathrm{NaCl}$ (face 100$)$ de taille $(3 \times 3 \mathrm{~mm})$. Après dissolution du support et flottage, les feuilles métalliques sont recueillies sur des grilles de cuivre électroformées de 200 mesh, ayant une transparence de $37 \%$.

L'épaisseur est contrôlée à l'aide d'une microbalance à quartz piézoélectrique construite dans 
notre laboratoire, dont le schéma de principe est donné par la figure 1. La variation $(\Delta F)$ de la fréquence $F=F_{1}-F_{2}$ (voir figure) est proportionnelle à l'épaisseur $e$ vaporisée, soit $e=-K \Delta F$. L'étalonnage a été effectué directement sur le monocristal de $\mathrm{NaCl}$ à l'aide d'un microscope interférentiel, pour une position donnée de la source, du quartz $\mathrm{Q}_{1}$ et du cristal de $\mathrm{NaCl}$. La valeur de $K$ obtenue pour l'aluminium est $0,56 \pm 0,06 \AA / \mathrm{Hz}$. Nous avons choisi ce procédé de mesure, car il permet de contrôler l'épaisseur en cours d'évaporation et d'obtenir ainsi d'une manière reproductible des couches minces d'épaisseur connue avec une incertitude absolue de $10 \%$, dans un domaine compris entre $50 \AA$ et $3000 \AA$.

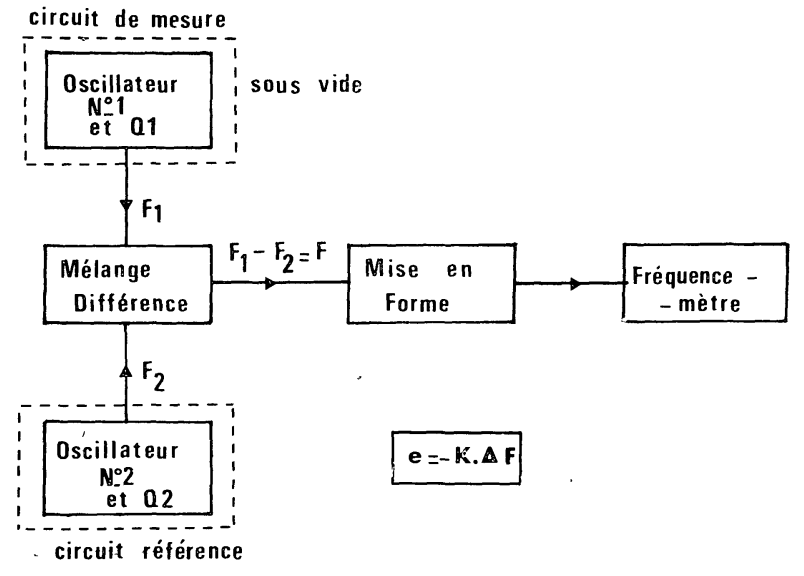

FIG. 1. - Schéma de principe de la microbalance à quartz piézoélectrique.

3. Dispositif expérimental. - Le dispositif expérimental, représenté figure 2 , comporte un groupe de pompage classique, avec piège à azote liquide, permettant d'atteindre au voisinage de l'objet une pression de l'ordre de $5 \times 10^{-6}$ torr. Deux canons à ions sont utilisables : un canon à ions gazeux (ions de gaz rares) et un canon à ions à source solide (ions alcalins). Les ions issus du canon sont accélérés sous quelques kilovolts et focalisés au moyen d'une lentille à 3 électrodes sur la surface de la feuille

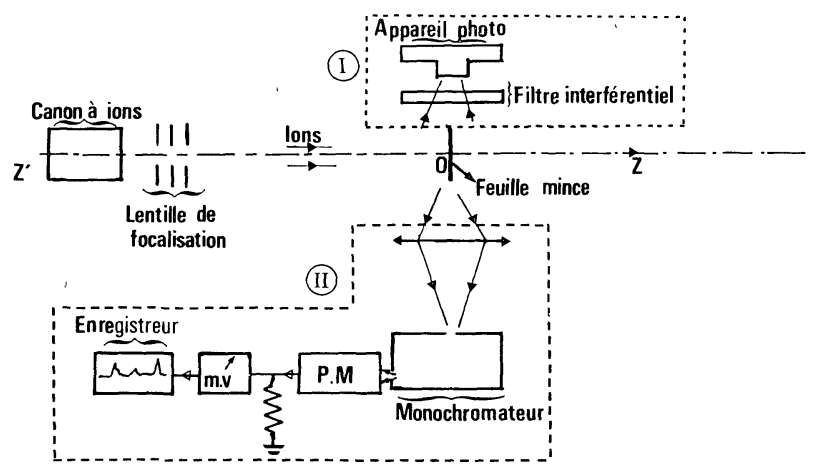

FIG. 2. - Schéma du montage expérimental. I) Dispositif d'enregistrement photographique. II) Dispositif d'enregistrement spectroscopique. mince constituant la cible. Un porte-objet tournant à 4 positions permet d'interposer successivement sous le faisceau 3 cibles différentes et une cage de mesure du débit ionique incident. Chaque cible est maintenue par 2 mors en laiton aux bords effilés.

Le schéma de la figure 3 représente le montage électrique de la cage de mesure du courant ionique.

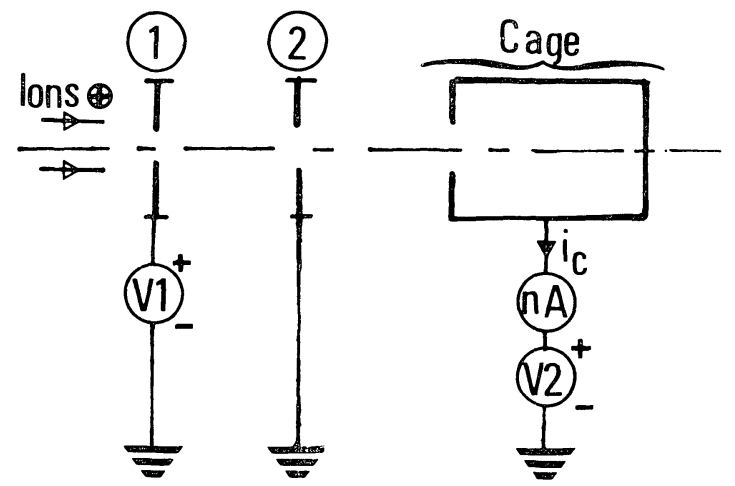

Fig. 3. - Cage de mesure. I) Diaphragme d'entrée d'ouverture Ø $2 \mathrm{~mm}$. II) Electrode de répulsion des électrons secondaires.

$$
V_{1}=V_{2}=70 \mathrm{~V} \text {. }
$$

Deux hublots en quartz dont les axes sont perpendiculaires à la direction du faisceau permettent d'observer et d'enregistrer les phénomènes lumineux au niveau de la cible. Le dispositif I se compose d'un appareil photographique utilisant un film à très haute sensibilité (1000 Asa) et enregistrant l'image des zones luminescentes après interposition sur le trajet lumineux d'un filtre interférentiel à bande étroite.

Le dispositif II comporte essentiellement un monochromateur à réseau de dispersion $33 \AA / \mathrm{mm}$ permettant d'explorer un domaine spectral compris entre 2000 et $6000 \AA$, suivi d'un photomultiplicateur à gain 'élevé sensible dans l'ultra-violet (EMI 6256, photocathode S 13).

4. Mise en évidence du phénomène. - La feuille d'aluminium sépare l'espace en 2 domaines $\mathbf{A}$ et $\mathbf{B}$ (Fig. 4), le domaine A étant placé du côté du bombardement.

Dans une première expérience nous avons bombardé une cible d'aluminium d'épaisseur $e=200 \AA$ au moyen d'ions $\mathrm{Na}^{+}$de $17 \mathrm{keV}$, la densité du faisceau étant de $40 \mu \mathrm{A} / \mathrm{cm}^{2}$.

Dans ces conditions, en l'absence de tout filtre interférentiel, nous avons réalisé une photographie de profil de la cible. Nous constatons (photo 1) qu'il a y bien émission de lumière de chaque côté de la couche bombardée.

Une analyse spectrographique effectuée suivant un axe perpendiculaire à la direction du bombardement (spectres Fig. $5 a, 5 b$ ), indique la présence de part et d'autre de la cible de radiations caractéristiques d'éléments provenant de l'échantillon bom- 


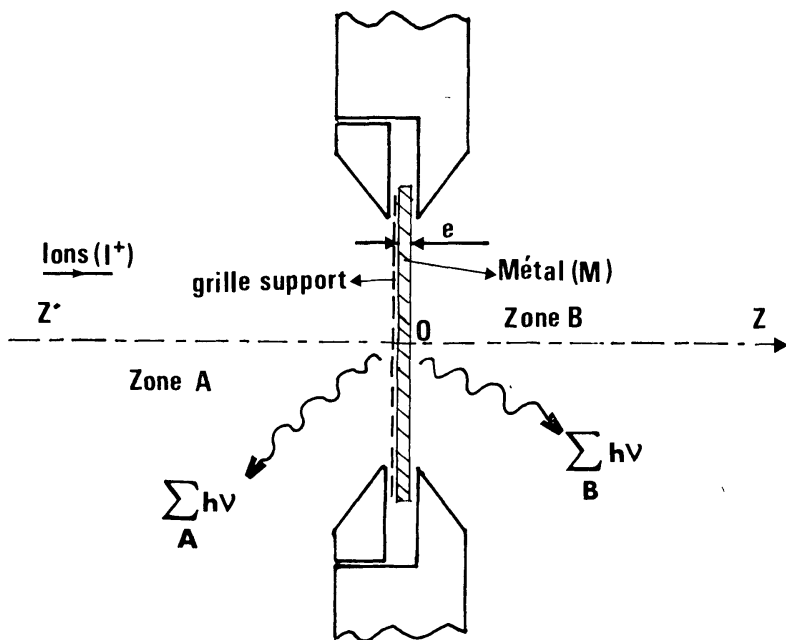

Fig. 4. - Positionnement de la cible. $\sum_{\mathbf{A}} h v$ : ensemble des radiations émises dans le domaine A. $\sum_{\mathbf{B}}^{\mathbf{A}} h v$ : ensemble des radiations émises dans le domaine $\mathrm{B}$.

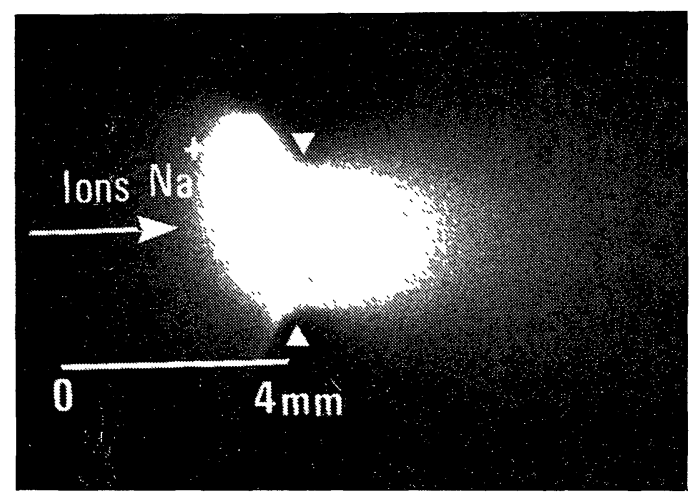

Photo. 1. - Image obtenue de profil non filtrée pour une cible de $200 \AA$ bombardée par des ions $\mathrm{Na}^{+}$de $17 \mathrm{keV}$.

bardé (Al I) ainsi que de radiations caractéristiques des éléments présents dans le faisceau ( $\mathrm{Na} \mathrm{I}$ ).

On remarque aussi la présence d'un élément présent dans le porte-objet et sur la grille $(\mathrm{Cu} \mathrm{I})$.

5. Analyse spectrale par la méthode photographique. - La possibilité d'interposer un filtre interférentiel nous permet d'obtenir des images filtrées dans un domaine spectral de $100 \AA$ environ. De ces photographies nous déduisons des renseignements globaux concernant l'anisotropie du phénomène et nous pouvons ainsi dégager l'influence des principaux paramètres.

En effet, si nous plaçons successivement ùn filtre centré sur $3960 \AA$ correspondant aux raies d'émission de l'aluminium $\lambda=3961 \AA$ et $\lambda=3944 \AA$, puis le filtre $\lambda=5890 \AA$ laissant passer le doublet du sodium, nous obtenons dans le cas d'une cible d'Al de $200 \AA$ bombardée par des ions $\mathrm{Na}^{+}\left(40 \mu \mathrm{A} / \mathrm{cm}^{2}, 17 \mathrm{keV}\right)$, les deux photographies filtrées (photos $2 a$ et $2 b$ ).

Nous remarquons tout d'abord que les zones
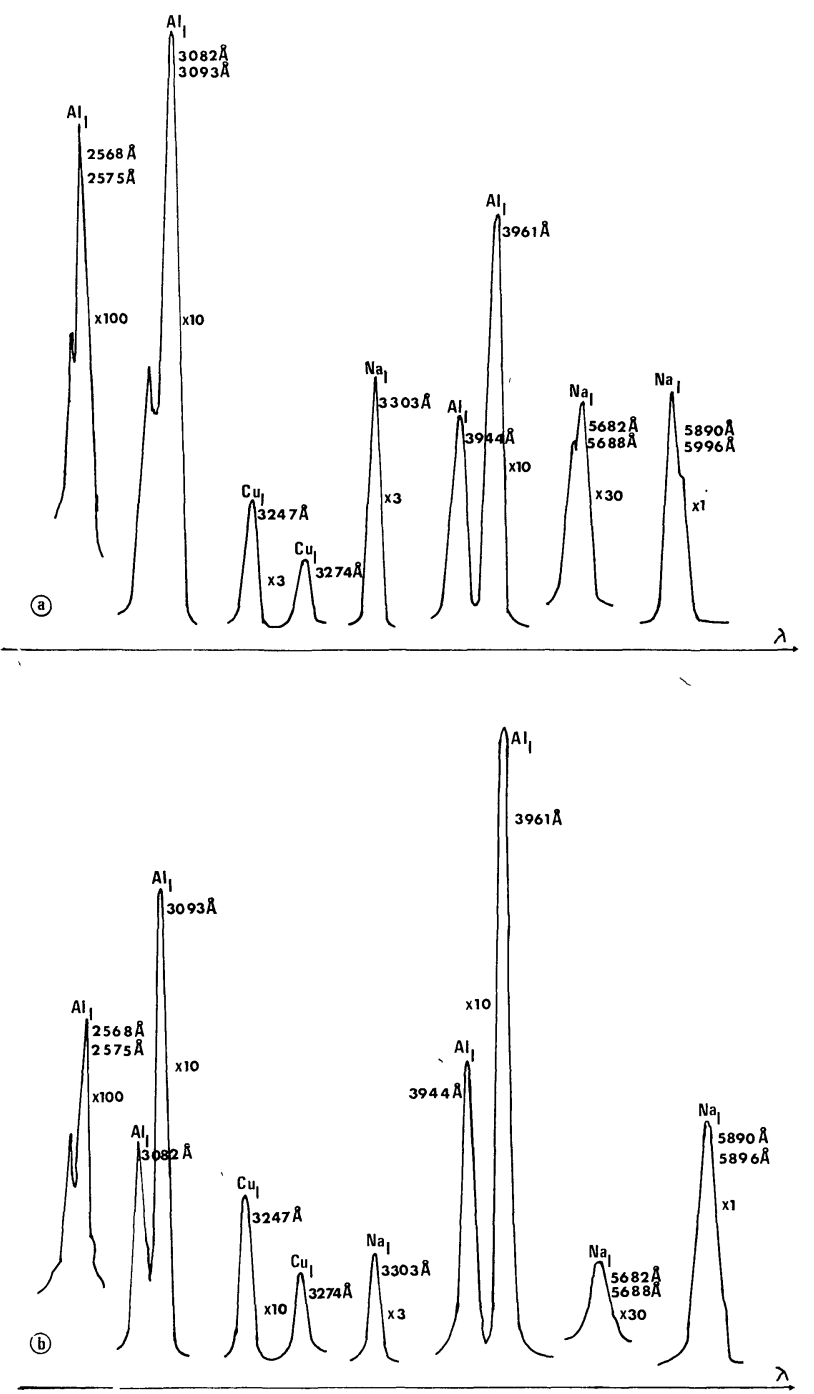

FIG. 5. - Spectres d'émission de la lumière dans les régions $\mathrm{A}$ et $\mathrm{B}, \grave{\mathrm{a}} \pm 100 \mu$ de la cible $(500 \AA)$. Ions $\mathrm{Na}^{+}$de $17 \mathrm{keV}$, densité $40 \mu \mathrm{A} / \mathrm{cm}^{2} ; a$ ) dans la région $\mathrm{A} ; b$ ) dans la région $\mathrm{B}$.

lumineuses sont beaucoup plus étendues dans le cas de l'image sodium que dans le cas de l'image aluminium. Ce phénomène s'explique, compte tenu du fait que les durées de vie des états excités correspondants sont peu différentes (16 ns pour $\mathrm{Na}, 7 \mathrm{~ns}$ pour $\mathrm{Al}$ ), par une énergie moyenne des atomes excités beaucoup plus élevée pour le sodium que pour l'aluminium. Ce résultat est une conséquence directe de la grande vitesse des ions primaires incidents $\mathrm{Na}^{+}$ et de l'immobilité initiale des atomes d'aluminium. D'autre part la répartition spatiale de l'émission lumineuse est fortement anisotrope et différente de part et d'autre de la cible.

Pour mettre en évidence l'influence de la nature de l'ion, nous avons bombardé des cibles d'Al, d'épaisseurs identiques $(e=200 \AA)$, successivement avec des ions $\mathrm{Li}^{+}, \mathrm{Na}^{+}$et $\mathrm{Ar}^{+}$(photo $3 a, b, c$ ) et observé l'émission de l'aluminium. Nous constatons que l'émission est d'autant plus intense et étendue que la masse de l'ion augmente. 


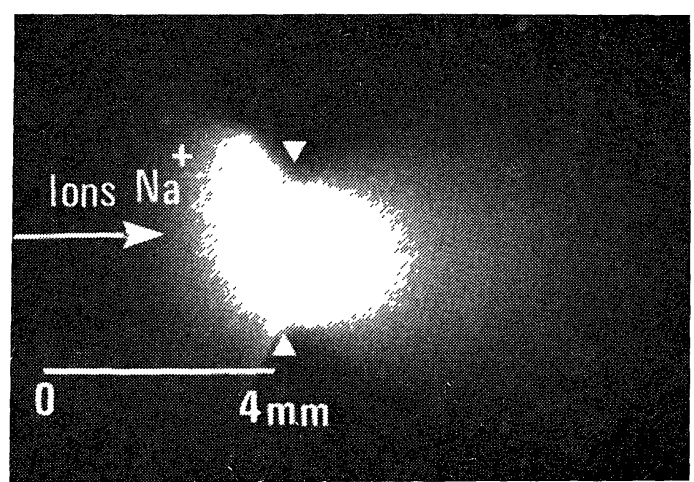

(a)

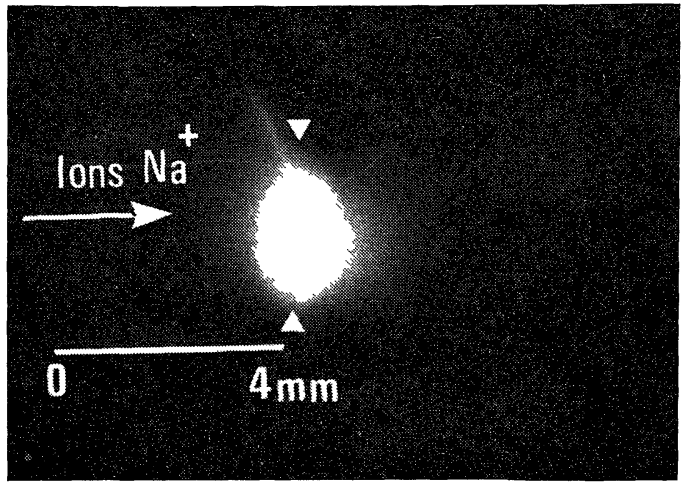

(b)

Рното. 2. - Images filtrées pour une cible de $200 \AA$ bombardée par des ions $\mathrm{Na}^{+}$de $17 \mathrm{keV}$. a) Image Na obtenue par filtrage des raies $5889 \AA$ et $5890 \AA$. b) Image Al obtenue par filtrage des raies $3961 \AA$ et $3944 \AA$.

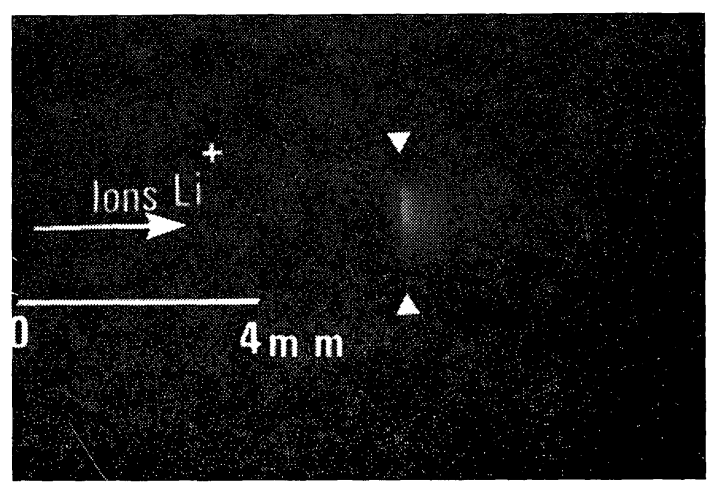

(a)

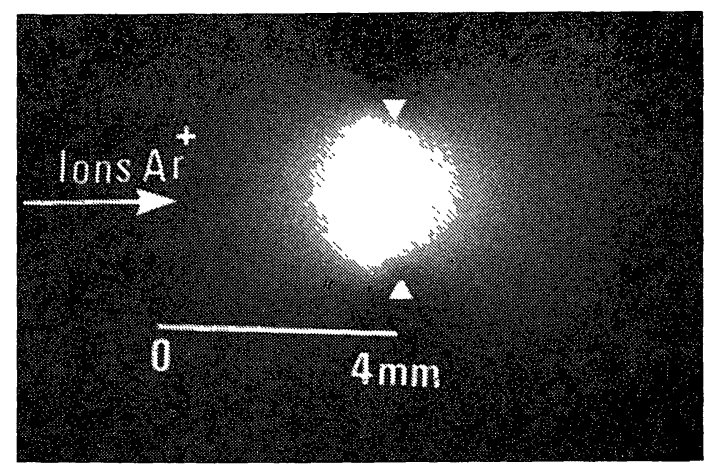

(c)

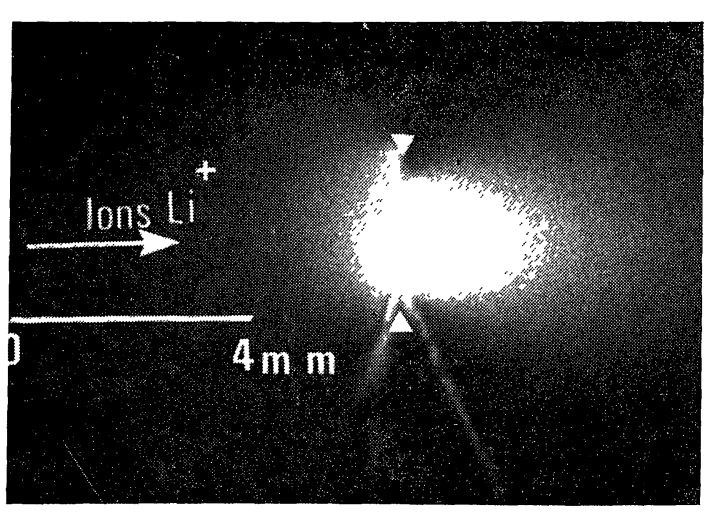

(a)

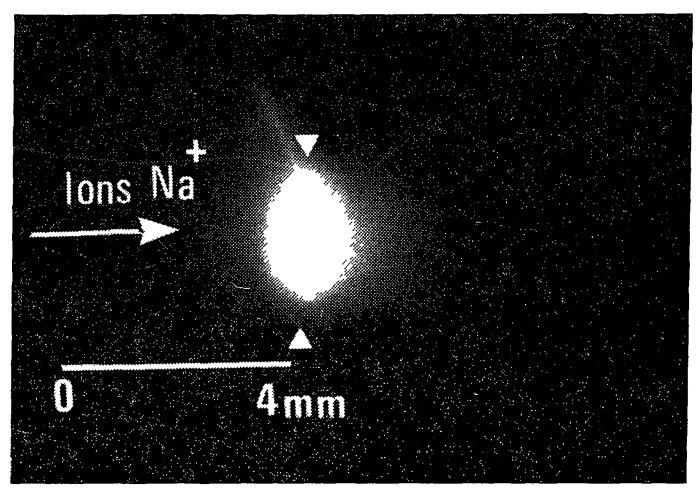

(b)

Рното. 3. - Poches lumineuses caractéristiques de l'aluminium obtenues par filtrage des raies $3961 \AA$ et $3944 \AA$ pour différents ions incidents. a) Ions $\mathrm{Li}^{+} 17 \mathrm{keV}$. b) Ions $\mathrm{Na}^{+} 17 \mathrm{keV}$. c) Ions

$$
\mathrm{Ar}^{+} 17 \mathrm{keV} ; e=200 \AA \text {. }
$$

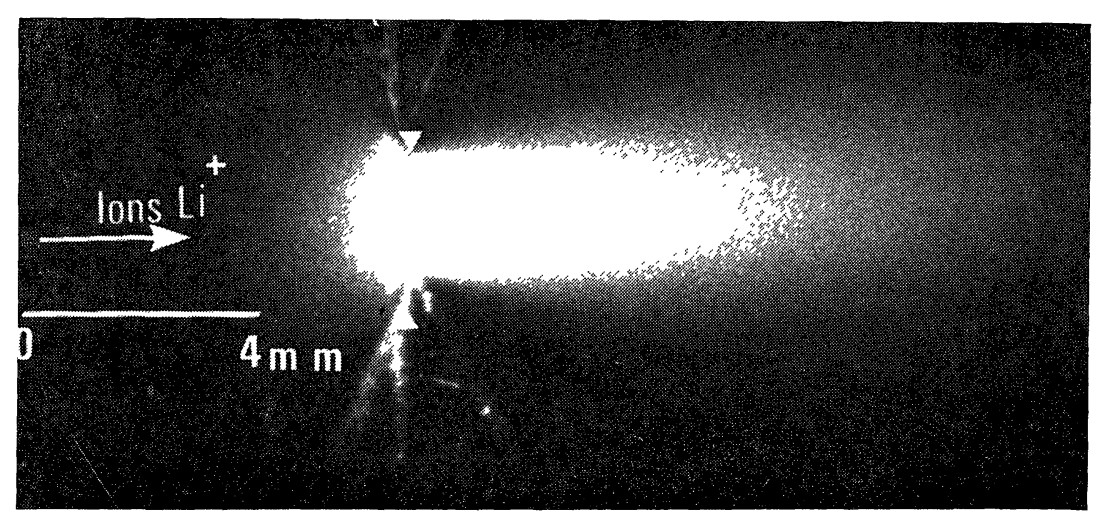

(b)

Pното. 4. - Poches lumineuses caractéristiques, du faisceau primaire d'ions $\mathrm{Li}^{+}$(raie $6104 \AA$ ) de $17 \mathrm{keV}$ pour deux épaisseurs de la cible. a) $e=400 \AA$. b) $e=200 \AA$. 
Enfin l'influence de l'épaisseur a été observée au cours de deux séries d'expériences sur des cibles d'Al d'épaisseurs différentes. Dans une première série, les ions incidents sont des ions $\mathrm{Li}^{+}$et on observe l'émission des atomes excités de $\mathrm{Li}$ (photo $4 a, b$ ). Il apparaît très nettement que l'influence de l'épaisseur de la cible est beaucoup moins marquée dans le domaine $\mathrm{A}$ que dans le domaine $\mathrm{B}$, où la trace lumineuse est d'autant plus étendue que l'épaisseur devient plus faible.

Nous arrivons aux mêmes conclusions au cours d'une deuxième série d'expériences (photos 5 et $3 b$ ) en bombardant successivement au moyen d'ions $\mathrm{Na}^{+}$des cibles d'aluminium d'épaisseur $400 \AA$ et $200 \AA$.

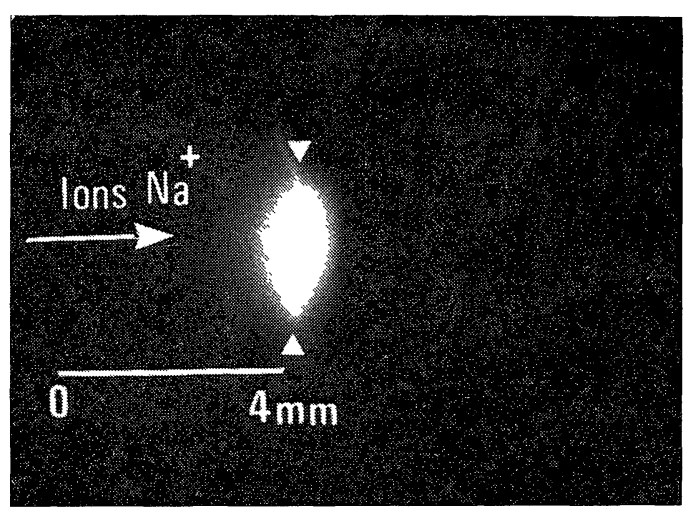

Рното. 5. - Poches lumineuses caractéristiques de la cible d'aluminium (raies $3961 \AA$ et $3944 \AA$ ) pour une épaisseur de $400 \AA$, bombardée par des ions $\mathrm{Na}^{+}$de $17 \mathrm{keV}$.

6. Analyse spectroscopique. - 6.1 EXPÉRIENCE PRÉLIMINAIRE: - Dans une première expérience nous avons bombardé une feuille d'aluminium de $600 \AA$ d'épaisseur au moyen d'un faisceau d'ions $\mathrm{Na}^{+}$de $17 \mathrm{keV}$ avec une densité de $40 \mu \mathrm{A} / \mathrm{cm}^{2}$. Nous avons étudié suivant l'axe $\mathrm{z}^{\prime}$ oz l'évolution, en fonction du temps, de l'intensité de la radiation $3961 \AA$ de Al I.

La courbe représentée figure 6 nous montre tout

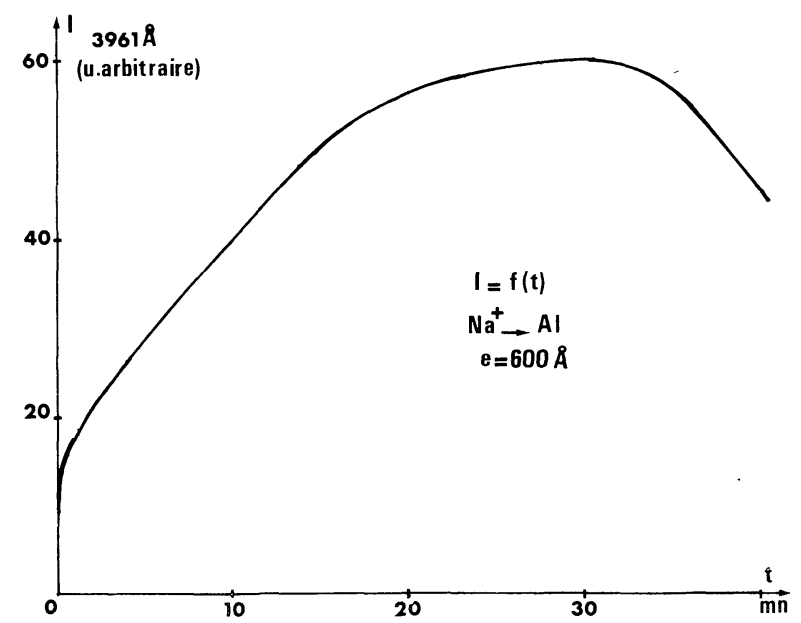

Fig. 6. - Evolution en fonction du temps de l'intensité de la radiation $3961 \AA$ de $\mathrm{AlI} \mathrm{I}$, pour une feuille de $600 \AA$. Ions $\mathrm{Na}^{+}$ de $17 \mathrm{keV}$, densité $40 \mu \mathrm{A} / \mathrm{cm}^{2}$. d'abord que l'intensité augmente régulièrement pendant $30 \mathrm{mn}$ environ. Cette augmentation s'explique par l'amincissement progressif de la couche d'aluminium, par pulvérisation, au cours du bombardement ionique. Nous remarquons ensuite une décroissance rapide de l'intensité, correspondant à la destruction des dernières couches monoatomiques constituant la cible.

L'émission de lumière est donc perturbée par le phénomène de pulvérisation. Néanmoins les résultats peuvent être reproductibles si on limite la durée d'observation de la luminescence d'une couche à 2 ou $3 \mathrm{mn}$ environ.

6.2 VARIATION DE L'INTENSITÉ D'UNE RADIATION DONNÉE LE LONG DE LA DIRECTION DE BOMBARDEMENT. - Le dispositif utilisé est celui représenté sur la figure 2 (dispositif II). L'observation est-faite de profil, dans une direction perpendiculaire à l'axe $\mathrm{z}^{\prime} \mathrm{oz}$.

On forme, au grandissement 1, l'image des zones lumineuses sur la fente d'entrée du monochromateur, au moyen d'une lentille en quartz de $50 \mathrm{~mm}$ de focale. Par déplacement de la lentille dans son plan et suivant une direction parallèle à $\mathrm{z}^{\prime} \mathrm{oz}$, on analyse de façon continue et le long de cet axe, une tranche d'épaisseur $\Delta z$ de la zone lumineuse correspondant à la largeur des fentes du monochromateur (Fig. 7).

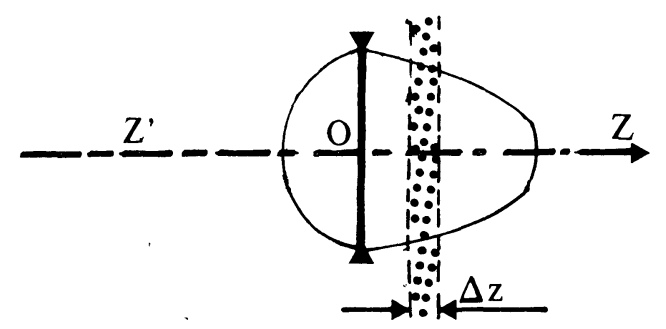

FIG. 7. - Schéma de principe montrant la zone de largeur $\Delta \mathbf{z}$ de lumière analysée par le monochromateur.

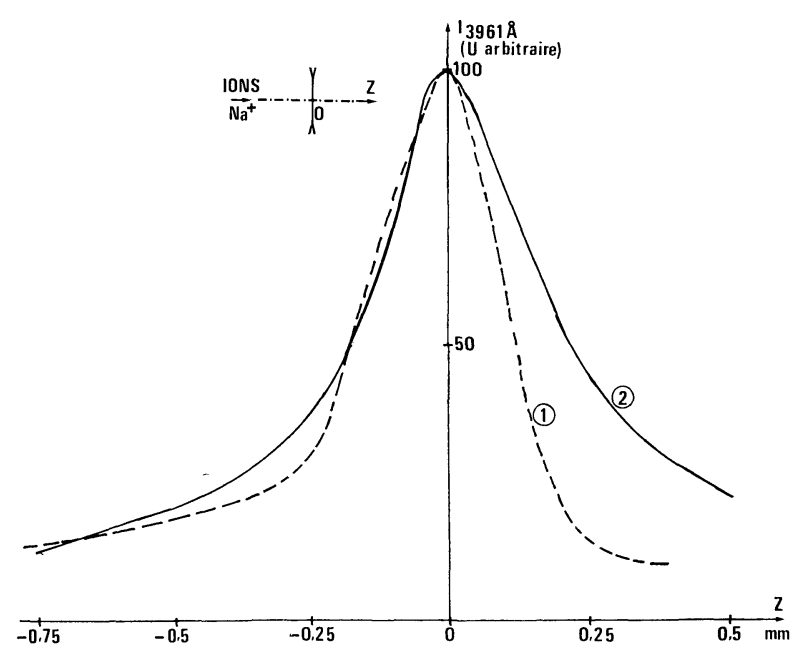

FIG. 8. - Evolution de l'intensité de la raie $3961 \AA$ de Al I le long de l'axe $\mathrm{z}^{\prime} \mathrm{oz}$. L'abscisse $\mathrm{O}$ correspondant à la cible. Ions $\mathrm{Na}^{+}$de $17 \mathrm{keV}$, densité $40 \mu \mathrm{A} / \mathrm{cm}^{2}$. 1) Cas d'une feuille d'aluminium d'épaisseur $1000 \AA$. 2) Cas d'une feuille d'aluminium d'épaisseur $300 \AA$. 
Nous avons bombardé des cibles d'aluminium d'épaisseurs différentes au moyen d'ions $\mathrm{Na}^{+}$de $17 \mathrm{keV}$, la densité du faisceau étant de $40 \mu \mathrm{A} / \mathrm{cm}^{2}$. La figure 8 représente l'enregistrement de la radiation $3961 \AA$ du spectre I de l'aluminium pour 2 épaisseurs de la cible (300 $\AA$ et $1000 \AA)$. On, constate que l'émission d'atomes excités provenant de la cible ne varie pas en fonction de l'épaisseur dans la zone A. Dans la zone B par contre, la longueur suivant $\mathrm{z}^{\prime} \mathrm{oz}$ de la région lumineuse augmente quand l'épaisseur diminue. Ceci peut s'expliquer par le fait que l'émission de lumière dans la région $\mathrm{A}$ n'intéresse que les premières couches monoatomiques comme pour les échantillons massifs, alors que l'émission dans la région $B$ est fortement influencée par l'épaisseur de la couche.

6.3 ETUDE PAR EFFET DOPPLER DES VITESSES D'ÉMISSION. - Pour cette dernière étude nous avons utilisé le même dispositif d'analyse que précédemment, les conditions expérimentales restant les mêmes. En différents points de l'axe $\mathrm{z}^{\prime}$ oz nous avons enregistré successivement deux raies caractéristiques de l'aluminium $(\lambda=3093 \AA)$ et $\mathrm{du}$ sodium $(\lambda=5890 \AA)$; puis mesuré la largeur à mi-hauteur de ces raies spectrales (Fig. 9). Les courbes de la figure 9 représentent également, du fait de la symétrie $\mathrm{du}$ système, la variation de l'effet Doppler le long de $z^{\prime}$ oz.

Nous constatons que les largeurs spectrales passent par un minimum au voisinage de la cible et présentent un maximum dans les 2 zones d'observation, celui-ci étant particulièrement visible dans le cas du sodium. Ces résultats indiquent suivant les hypothèses faites par Mériaux [2], la présence de chaque côté de la cible de deux distributions de vitesses des atomes émis à l'état excité.

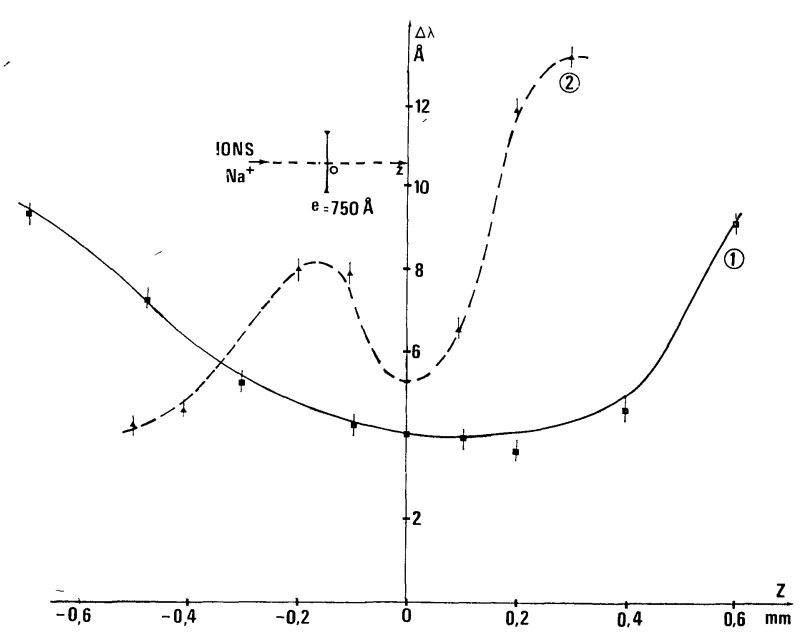

FIG. 9. - Evolution de la largeur à mi-hauteur des radiations $3093 \AA$ de Al I (courbe 1) et 5890 de Na I (courbe 2) le long de $z^{\prime}$ oz, épaisseur de la couche d'aluminium $750 \AA$. Ions $\mathrm{Na}^{+}$ de $17 \mathrm{keV}$, densité $40 \mu \mathrm{A} / \mathrm{cm}^{2}$.

7. Conclusion. - Ce travail a mis en évidence le fait que sous l'impact d'ions positifs de quelques kiloélectronvolts une feuille mince émet des particules excitées, non seulement du côté du faisceau ionique incident, comme dans le cas des échantillons massifs, mais également du côté opposé au bombardement.

Les moyens d'étude utilisés, nous permettent de tirer déjà quelques conclusions sur l'influence de l'épaisseur et sur la distribution des vitesses des particules pulvérisées ou diffusées, mais toutefois ces mesures demanderont à être complétées par d'autres expériences en cours. En particulier, nous comptons développer l'étude, en fonction de l'épaisseur, des distributions de vitesses des atomes excités au moyen de l'effet Doppler et également préciser l'influence de la nature de la cible sur ce phénomène.

\section{Bibliographie}

[1] Meriaux, Thèse doctorat ès Sciences Physiques, 1971, Lyon.

[2] MeriauX, J. P., Goutte, R. et Guillaud, C., IVe Symposium international sur les décharges et l'isolement électrique dans le vide, Waterloo, Canada 1970. 